Journal of Universal Mathematics

VOL.2 No.1 PP.42-50 (2019)

ISSN-2618-5660

\title{
ON DIGITAL H-GROUPS
}

\author{
S.DEMIRALP AND ESSAM H. HAMOUDA
}

\begin{abstract}
In this paper, specific properties of digital $\mathrm{H}$-spaces and digital $\mathrm{H}$-groups are studied. It is shown that there is a contravariant functor from the homotopy category of the pointed digital images to the category of groups and homomorphisms. Then it is proven that a pointed digital image having the same digital homotopy type as a digital H-group is itself a digital H-group.
\end{abstract}

\section{INTRODUCTION}

The purpose of digital topology is to study the topological properties of discrete objects, such as compactness, connectedness. Digital topology was first studied in [14] by the computer image analysis researcher Rosenfeld. Following years researchers studied the digital versions of many concepts of algebraic topology. Digital homotopy and digital fundamental group are defined in [4] by Boxer. Digital $\mathrm{H}$-space is defined in [7] by Ege and Karaca. H-spaces are an important concept of homotopy theory. An H-space consists of a pointed topological space $\mathrm{P}$ with a continuous multiplication $m: X \times X \rightarrow X$ and with a constant map $c: X \rightarrow X$, such that $m \circ\left(c, 1_{X}\right) \simeq 1_{X} \simeq m \circ\left(1_{X}, c\right)$. A group structure can be established on $\mathrm{H}$-space, called H-group, by homotopy group operations which are similar to group operations.

\section{Preliminaries}

Let $\mathbb{Z}$ be the set of all integers and $\mathbb{Z}^{n}$ the set of all lattice points in Euclidean n-dimensional space. A finite subset $X$ of $\mathbb{Z}^{n}$ with an adjacency relation $\kappa$ is called a digital image, denoted by $(X, \kappa)$.

For a positive integer $t$ with $1 \leq t \leq n$, two distinct points $x=\left(x_{1}, x_{2}, \ldots, x_{n}\right)$, $y=\left(y_{1}, y_{2}, \ldots, y_{n}\right) \in \mathbb{Z}^{n}$ are $\kappa_{t}$-adjacent if,

i) there are at most $t$ distinct indices $i$ such that $\left|x_{i}-y_{i}\right| \neq 1$, and

ii) for all indices $j$, if $\left|x_{j}-y_{j}\right| \neq 1$, then $x_{j}=y_{j}$.

Consider the following statements for the commonly used adjacency relations:

(1) $p, q \in \mathbb{Z}$ are 2-adjacent if $|p-q|=1$

Date: Review October 11, 2018, accepted January 26, 2019.

2000 Mathematics Subject Classification. Primary 55P45, 68R99; Secondary 55P65, 55P10.

Key words and phrases. Digital image, Digital homotopy, Digital H-space, Digital H-group. 
(2) $p, q \in \mathbb{Z}^{2}$ are 8 -adjacent if they are distinct and differ by at most 1 each coordinate.

(3) $p, q \in \mathbb{Z}^{2}$ are 4 -adjacent if they are 8-adjacent and differ by exactly one coordinate.

(4) $p, q \in \mathbb{Z}^{3}$ are 26-adjacent if they distinct and differ by at most 1 each coordinate.

(5) $p, q \in \mathbb{Z}^{3}$ are 18 -adjacent if they are 26 -adjacent and differ by at most two coordinates.

(6) $p, q \in \mathbb{Z}^{3}$ are 6 -adjacent if they are 18 -adjacent and differ by exactly one coordinate.

A digital interval is a set of the form $[a, b]_{\mathbb{Z}}=\{z \in \mathbb{Z} \mid a \leq z \leq b\}$.

The adjacency relation on cartesian product of two digital image is defined as follows.

Definition 2.1. [8] For two digital image $\left(X, \kappa_{1}\right)$ and $\left(Y, \kappa_{2}\right)$, the $\kappa^{*}$-adjacency on the product image $X \times Y$ is obtained as follows: $x_{1}, x_{2} \in\left(X, \kappa_{1}\right), y_{1}, y_{2} \in\left(Y, \kappa_{2}\right)$, then $\left(x_{1}, y_{1}\right)$ and $\left(x_{2}, y_{2}\right)$ are $\kappa^{*}$-adjacent if and only if one of the following is satisfied:

(1) $x_{1}=x_{2}$ and $y_{1}$ and $y_{2}$ are $\kappa_{2}$-adjacent,

(2) $x_{1}$ and $x_{2}$ are $\kappa_{1}$-adjacent and $y_{1}=y_{2}$,

(3) $x_{1}$ and $x_{2}$ are $\kappa_{1}$-adjacent and $y_{1}$ and $y_{2}$ are $\kappa_{2}$-adjacent.

Definition 2.2. [6] Let $\left(X, \kappa_{1}\right)$ and $\left(Y, \kappa_{2}\right)$ be digital images. Then the function $f: X \rightarrow Y$ is $\left(\kappa_{1}, \kappa_{2}\right)$-continuous if and only if for every $\left\{x_{0}, x_{1}\right\} \subset X$ such that $x_{0}$ and $x_{1}$ are $\kappa_{1}$-adjacent, either $f\left(x_{0}\right)=f\left(x_{1}\right)$ or $f\left(x_{0}\right)$ and $f\left(x_{1}\right)$ are $\kappa_{2}$-adjacent.

Example 2.3. Let $f: \mathbb{Z}^{2} \rightarrow \mathbb{Z}$ be defined as $f(x)=x_{1}+x_{2}$, for all $x=\left(x_{1}, x_{2}\right) \in$ $\mathbb{Z}^{2}$. Then it is clear that $f$ is $(4,2)$-continuous. However it isn't $(8,2)$-continuous, because $x=\left(x_{1}, x_{2}\right)$ and $y=\left(x_{1}+1, x_{2}+1\right)$ are 8 -adjacent but $f(x)=x_{1}+x_{2}$ and $f(y)=x_{1}+x_{2}+2$ are not 2 -adjacent.

Definition 2.4. [4] Let $\left(X, \kappa_{1}\right)$ and $\left(Y, \kappa_{2}\right)$ be two digital image and $f$ and $g$ be $\left(\kappa_{1}, \kappa_{2}\right)$-continuous functions. If

(1) there exist a function $F: X \times[0, m]_{\mathbb{Z}} \rightarrow Y$, such that, for all $x \in X$, $F(x, 0)=f(x)$ and $F(x, m)=g(x)$,

(2) the induced function $F_{x}:[0, m]_{\mathbb{Z}} \rightarrow Y, F_{x}(t)=F(x, t)$ for all $x \in X$ and for all $t \in[0, m]_{\mathbb{Z}}$, is $\left(2, \kappa_{2}\right)$-continuous and

(3) the induced function $F_{t}: X \rightarrow Y, F_{t}(x)=F(x, t)$ for all $x \in X$ and for all $t \in[0, m]_{\mathbb{Z}}$, is $\left(\kappa_{1}, \kappa_{2}\right)$-continuous,

then $f$ and $g$ are said to be digitally $\left(\kappa_{1}, \kappa_{2}\right)$-homotopic and $F$ is called a digital $\left(\kappa_{1}, \kappa_{2}\right)$-homotopy between $f$ and $g$, written $f \stackrel{F}{\simeq}_{\left(\kappa_{1}, \kappa_{2}\right)} g$ (or $f \simeq_{\left(\kappa_{1}, \kappa_{2}\right)} g$, for short).

The notation $[f]$ is used to denote the digital homotopy class of $\left(\kappa_{1}, \kappa_{2}\right)$-continuous function $f: X \rightarrow Y$, i.e.

$$
[f]=\left\{g: X \rightarrow Y \mid \mathrm{g} \text { is }\left(\kappa_{1}, \kappa_{2}\right) \text {-continuous and } f \simeq_{\left(\kappa_{1}, \kappa_{2}\right)} g\right\} .
$$

The set of all digital homotopy classes of $\left(\kappa_{1}, \kappa_{2}\right)$-continuous functions is denoted by $\left[\left(X, \kappa_{1}\right),\left(Y, \kappa_{2}\right)\right]$, i.e.

$$
\left[\left(X, \kappa_{1}\right),\left(Y, \kappa_{2}\right)\right]=\left\{[f] \mid f:\left(X, \kappa_{1}\right) \rightarrow\left(Y, \kappa_{2}\right) \text { is }\left(\kappa_{1}, \kappa_{2}\right) \text {-continuous }\right\} .
$$


For a digital image $(X, \kappa)$ and its subset $(A, \kappa),(X, A, \kappa)$ is called a digital image pair with $\kappa$-adjacency. Also, if $A$ is a singleton set $\{p\}$, then $(X, p, \kappa)$ is called a pointed digital image.

Definition 2.5. [4] Let $\left(X, \kappa_{1}\right)$ and $\left(Y, \kappa_{2}\right)$ be two digital image and $f$ be a $\left(\kappa_{1}, \kappa_{2}\right)$ continuous function and $g$ be a $\left(\kappa_{2}, \kappa_{1}\right)$-continuous function, such that

$$
f \circ g \simeq_{\left(\kappa_{2}, \kappa_{2}\right)} 1_{Y} \text { and } g \circ f \simeq_{\left(\kappa_{1}, \kappa_{1}\right)} 1_{X} .
$$

Then $\left(X, \kappa_{1}\right)$ and $\left(Y, \kappa_{2}\right)$ are said to be same $\left(\kappa_{1}, \kappa_{2}\right)$-homotopy type or $\left(\kappa_{1}, \kappa_{2}\right)$ homotopy equivalent. Also $f$ and $g$ are called $\left(\kappa_{1}, \kappa_{2}\right)$-equivalences.

\section{Digital H-spaces}

In this section some properties of digital $\mathrm{H}$-spaces and digital $\mathrm{H}$-groups are investigated. It is shown that the set of homotopy classes of digitally continuous functions, from a homotopy associative digital $\mathrm{H}$-space to a pointed digital image, is semigroup. Then it is proven that a pointed digital image having the same homotopy type as an abelian digital H-group is itself an abelian digital H-group. Also it is shown that there is a contravariant functor from the homotopy category of the pointed digital images to the category of abelian groups and homomorphisms.

Definition 3.1. [7] Let $(X, p, \kappa)$ be a pointed digital image. For a digital continuous multiplication $\mu: X \times X \rightarrow X$ and the digital constant map $c: X \rightarrow X$, defined by $c(x)=p$, if $\mu \circ\left(c, 1_{X}\right) \simeq_{(\kappa, \kappa)} \mu \circ\left(1_{X}, c\right) \simeq_{(\kappa, \kappa)} 1_{X}$, then $(X, p, \kappa)$ is called a digital $\mathrm{H}$-space.

Example 3.2. Let $(\mathbb{Z}, 0,2)$ be pointed digital image and let $\mu: \mathbb{Z} \times \mathbb{Z} \rightarrow \mathbb{Z}$ defined as $\mu(x, y)=x+y$. It is clear that $\mu$ is $(4,2)$-continuous.

$$
\begin{aligned}
& \mu \circ\left(c, 1_{X}\right)(x)=\mu\left(c(x), 1_{X}(x)\right)(x)=\mu(0, x)=0+x=x \\
& \mu \circ\left(1_{X}, c\right)(x)=\mu\left(1_{X}(x), c(x)\right)(x)=\mu(x, 0)=x+0=x
\end{aligned}
$$

where $c: \mathbb{Z} \rightarrow \mathbb{Z}$ is a constant map, $c(x)=0$. Therefore

$$
\mu \circ\left(c, 1_{X}\right) \simeq_{(2,2)} 1_{X} \simeq_{(2,2)} \mu \circ\left(1_{X}, c\right) .
$$

Consequently $(\mathbb{Z}, 0,2)$ is a digital $\mathrm{H}$-space.

Definition 3.3. [7] Let $(X, p, \kappa)$ be a digital H-space. If

$$
\mu \circ\left(1_{X} \times \mu\right) \simeq_{\left(\kappa^{*}, \kappa\right)} \mu \circ\left(\mu \times 1_{X}\right)
$$

then $\mu$ is called digital homotopy associative.

If there exists a map $\eta:(X, p, \kappa) \rightarrow(X, p, \kappa)$ such that,

$$
\mu \circ\left(\eta, 1_{X}\right) \simeq_{(\kappa, \kappa)} \mu \circ\left(1_{X}, \eta\right) \simeq_{(\kappa, \kappa)} c
$$

then $\eta$ is called a digital homotopy inverse for $\mu$.

If there exists a map $T: X \times X \rightarrow X \times X, T(x, y)=(y, x)$, such that

$$
\mu \circ T \simeq_{\left(\kappa^{*}, \kappa\right)} \mu
$$

then $\mu$ is called digital homotopy commutative and $(X, p, \kappa)$ is called abelian digital $\mathrm{H}$-space.

Theorem 3.4. Let $\left(X, p, \kappa_{1}\right)$ be a digital H-space with digital homotopy associative multiplication $\mu$ and $\left(Y, q, \kappa_{2}\right)$ be a pointed digital image. Then $\left[\left(Y, q, \kappa_{2}\right),\left(X, p, \kappa_{1}\right)\right]$ is a semigroup with identity. 
Proof. For any $[f],[g] \in\left[\left(Y, q, \kappa_{2}\right),\left(X, p, \kappa_{1}\right)\right]$, let define the product

$$
[f] \bullet[g]=[\mu \circ(f \times g) \circ \triangle]
$$

where $\triangle: Y \rightarrow Y \times Y, \triangle(y)=(y, y)$.Let $[f]=\left[f^{\prime}\right]$ and $[g]=\left[g^{\prime}\right]$, then there exist $\left(\kappa_{2}, \kappa_{1}\right)$-homotopies $H$ and $G$ such that $f \stackrel{G}{\simeq}_{\left(\kappa_{2}, \kappa_{1}\right)} f^{\prime}$ and $g \stackrel{H}{\simeq}\left(\kappa_{2}, \kappa_{1}\right) g^{\prime}$. Define a digital homotopy $F: Y \times[0, m]_{\mathbb{Z}} \rightarrow X$ as $F=\mu \circ(G, H)$. Then

$$
\begin{aligned}
F(y, 0) & =\mu \circ(G, H)(y, 0) \\
& =\mu(G(y, 0), H(y, 0)) \\
& =\mu(f(y), g(y)) \\
& =(\mu \circ(f \times g) \circ \triangle)(y)
\end{aligned}
$$

and similarly $F(y, m)=\left(\mu \circ\left(f^{\prime} \times g^{\prime}\right) \circ \triangle\right)(y)$. So

$$
(\mu \circ(f \times g) \circ \triangle) \stackrel{F}{\simeq}_{\left(\kappa_{2}, \kappa_{1}\right)}\left(\mu \circ\left(f^{\prime} \times g^{\prime}\right) \circ \triangle\right) .
$$

Then

$$
\begin{aligned}
{[f] \bullet[g] } & =[\mu \circ(f \times g) \circ \triangle] \\
& =\left[\mu \circ\left(f^{\prime} \times g^{\prime}\right) \circ \triangle\right] \\
& =\left[f^{\prime}\right] \bullet\left[g^{\prime}\right] .
\end{aligned}
$$

Consequently" •" is well defined.

Let $c:\left(X, p, \kappa_{1}\right) \rightarrow\left(X, p, \kappa_{1}\right)$ be the constant map $c(x)=p, \forall x \in X$. Let define a map $e:\left(Y, q, \kappa_{2}\right) \rightarrow\left(X, p, \kappa_{1}\right)$ such that $e(y)=p$, for all $y \in Y$. Then

$$
(\mu \circ(e \times f) \circ \triangle)(y)=\mu(p, f(y))=\left(\mu \circ\left(c, 1_{X}\right) \circ f\right)(y)
$$

and since $\mu \circ\left(c, 1_{X}\right) \simeq_{\left(\kappa_{1}, \kappa_{1}\right)} 1_{X}$, then $[e] \bullet[f]=[\mu \circ(e \times f) \circ \Delta]=[f]$. So $[e]$ is the identity element of $\left[\left(Y, q, \kappa_{2}\right),\left(X, p, \kappa_{1}\right)\right]$.

Let $[f],[g],[h] \in\left[\left(Y, q, \kappa_{2}\right),\left(X, p, \kappa_{1}\right)\right]$.

$$
\begin{aligned}
{[f] \bullet([g] \bullet[h]) } & =[f] \bullet[\mu \circ(g \times h) \circ \Delta] \\
& =[\mu \circ(f \times(\mu \circ(g \times h) \circ \triangle)) \circ \triangle] \\
& =\left[\mu \circ\left(1_{X} \times \mu\right) \circ(f \times g \times h) \circ\left(1_{X} \times \triangle\right) \circ \triangle\right] \\
& =\left[\mu \circ\left(\mu \times 1_{X}\right) \circ(f \times g \times h) \circ\left(1_{X} \times \triangle\right) \circ \triangle\right] \\
& =[\mu \circ((\mu \circ(f \times g) \circ \triangle) \times h) \circ \triangle] \\
& =([f] \bullet[g]) \bullet[h] .
\end{aligned}
$$

Therefore "•" is digital homotopy associative.

Theorem 3.5. [7] Let $\left(X, p, \kappa_{1}\right)$ be a digital H-space and $\left(Y, q, \kappa_{2}\right)$ be a pointed digital image. If $\left(X, p, \kappa_{1}\right)$ and $\left(Y, q, \kappa_{2}\right)$ have the same $\left(\kappa_{1}, \kappa_{2}\right)$-homotopy type, then $\left(Y, q, \kappa_{2}\right)$ is a digital H-space.

Definition 3.6. Let $\left(X, p, \kappa_{1}\right)$ and $\left(Y, q, \kappa_{2}\right)$ be digital H-spaces. A map $f: X \rightarrow Y$ is called a digital H-homomorphism if $f \circ \mu \simeq_{\left(\kappa_{1}, \kappa_{2}\right)} \eta \circ(f \times f)$, where $\eta: Y \times Y \rightarrow Y$.

Theorem 3.7. Let $\left(X, p, \kappa_{1}\right)$ be a digital H-space and $\left(Y, q, \kappa_{2}\right)$ have the same $\left(\kappa_{1}, \kappa_{2}\right)$-homotopy type with $X$. Then digital $\left(\kappa_{1}, \kappa_{2}\right)$-equivalences are digital $\mathrm{H}$ homomorphisms. 
Proof. Let $f: X \rightarrow Y$ be a $\left(\kappa_{1}, \kappa_{2}\right)$-continuous function and let $g: Y \rightarrow X$ be a $\left(\kappa_{2}, \kappa_{1}\right)$-continuous function, such that $f \circ g \simeq_{\left(\kappa_{2}, \kappa_{2}\right)} 1_{Y}$ and $g \circ f \simeq_{\left(\kappa_{1}, \kappa_{1}\right)} 1_{X}$. Let $\mu$ be $\left(\kappa^{*}, \kappa_{1}\right)$-continuous multiplication of $\left(X, p, \kappa_{1}\right)$, then $\left(Y, q, \kappa_{2}\right)$ is a digital $\mathrm{H}$-space with the $\left(\kappa^{*}, \kappa_{2}\right)$ - continuous multiplication $\eta=f \circ \mu \circ(g \times g)$. Then

$$
g \circ \eta=g \circ(f \circ \mu \circ(g \times g)) \simeq_{\left(\kappa^{*}, \kappa_{1}\right)} 1_{X} \circ \mu \circ(g \times g)=\mu \circ(g \times g) .
$$

So $g$ is a digital H-homomorphism. Also,

$$
\eta \circ(f \times f)=f \circ \mu \circ(g \times g) \circ(f \times f) \simeq_{\left(\kappa^{*}, \kappa_{2}\right)} f \circ \mu \circ 1_{X \times X}=f \circ \mu .
$$

Therefore $f$ is a digital H-homomorphism.

Definition 3.8. A digital $\mathrm{H}$-group is a digital $\mathrm{H}$-space $(X, p, \kappa)$ with the digital homotopy associative multiplication $\mu$ and digital homotopy inverse $\eta$.

It is clear that $(\mathbb{Z}, 0,2)$ in Example 7 is an abelian digital H-group.

Theorem 3.9. Let $\left(X, p, \kappa_{1}\right)$ be a digital H-group. If $\left(X, p, \kappa_{1}\right)$ and $\left(Y, q, \kappa_{2}\right)$ have the same $\left(\kappa_{1}, \kappa_{2}\right)$-homotopy type, then $\left(Y, q, \kappa_{2}\right)$ is a digital H-group.

Proof. Let $f: X \rightarrow Y$ be a $\left(\kappa_{1}, \kappa_{2}\right)$-continuous function and let $g: Y \rightarrow X$ be a $\left(\kappa_{2}, \kappa_{1}\right)$-continuous function, such that $f \circ g \simeq_{\left(\kappa_{2}, \kappa_{2}\right)} 1_{Y}$ and $g \circ f \simeq_{\left(\kappa_{1}, \kappa_{1}\right)} 1_{X}$. Let $\eta=f \circ \mu \circ(g \times g)$ be digital continuous multiplication of $\left(Y, q, \kappa_{2}\right)$ where $\mu: X \times X \rightarrow X$. Then $\left(Y, q, \kappa_{2}\right)$ is a digital H-space. Since $f \circ g \simeq_{\left(\kappa_{2}, \kappa_{2}\right)} 1_{Y}$,

$$
\begin{aligned}
& \eta \times 1_{Y}=(f \circ \mu \circ(g \times g)) \times 1_{Y} \simeq_{\left(\kappa^{*}, \kappa_{2}\right)}(f \times f) \circ\left(\mu \times 1_{X}\right) \circ(g \times g \times g) \\
& 1_{Y} \times \eta=1_{Y} \times(f \circ \mu \circ(g \times g)) \simeq_{\left(\kappa^{*}, \kappa_{2}\right)}(f \times f) \circ\left(1_{X} \times \mu\right) \circ(g \times g \times g) .
\end{aligned}
$$

As $\left(X, p, \kappa_{1}\right)$ is a digital H-group, $\mu$ is digital homotopy associative. Then,

$$
\begin{aligned}
\eta \circ\left(\eta \times 1_{Y}\right) & \simeq\left(_{\left(\kappa^{*}, \kappa_{2}\right)} f \circ \mu \circ(g \times g) \circ(f \times f) \circ\left(\mu \times 1_{X}\right) \circ(g \times g \times g)\right. \\
& \simeq\left(\kappa^{*}, \kappa_{2}\right) f \circ \circ 1_{X \times X} \circ\left(\mu \times 1_{X}\right) \circ(g \times g \times g) \\
& =f \circ\left(\mu \circ\left(\mu \times 1_{X}\right)\right) \circ(g \times g \times g) \\
& \simeq{ }_{\left(\kappa^{*}, \kappa_{2}\right)} f \circ\left(\mu \circ\left(1_{X} \times \mu\right)\right) \circ(g \times g \times g) \\
& =f \circ \mu \circ 1_{X \times X} \circ\left(1_{X} \times \mu\right) \circ(g \times g \times g) \\
& \simeq\left(\kappa^{*}, \kappa_{2}\right) f \circ \mu \circ(g \times g) \circ(f \times f) \circ\left(1_{X} \times \mu\right) \circ(g \times g \times g) \\
& \simeq\left(\kappa^{*}, \kappa_{2}\right) \eta \circ\left(1_{Y} \times \eta\right) .
\end{aligned}
$$

Therefore $\eta$ is digital homotopy associative.

Let $\theta:\left(X, p, \kappa_{1}\right) \rightarrow\left(X, p, \kappa_{1}\right)$ be digital homotopy inverse for $\mu$ and $\theta^{\prime}=f \circ \theta \circ g$. Then,

$$
\begin{aligned}
\eta \circ\left(\theta^{\prime}, 1_{Y}\right) & =(f \circ \mu) \circ(g \times g) \circ\left(\theta^{\prime}, 1_{Y}\right) \\
& =(f \circ \mu) \circ(g \times g) \circ\left(f \circ \theta \circ g, 1_{Y}\right) \\
& =(f \circ \mu) \circ\left(g \circ f \circ \theta \circ g, g \circ 1_{Y}\right) \\
& \simeq\left(\kappa_{2}, \kappa_{2}\right)(f \circ \mu) \circ\left(1_{X} \circ \theta \circ g, g\right) \\
& =f \circ\left(\mu \circ\left(\theta, 1_{X}\right)\right) \circ g \\
& \simeq\left(_{\left.\kappa_{2}, \kappa_{2}\right)} f \circ c \circ g\right. \\
& \simeq{ }_{\left(\kappa_{2}, \kappa_{2}\right)} c^{\prime},
\end{aligned}
$$

where $c^{\prime}:\left(Y, q, \kappa_{2}\right) \rightarrow\left(Y, q, \kappa_{2}\right), c^{\prime}(y)=q, \forall y \in Y$. Similarly, $\eta \circ\left(1_{Y} \circ \theta^{\prime}\right) \simeq_{\left(\kappa_{2}, \kappa_{2}\right)} c^{\prime}$. Hence $\theta^{\prime}$ is a homotopy inverse for $\left(Y, q, \kappa_{2}\right)$.

Consequently $\left(Y, q, \kappa_{2}\right)$ is a digital H-group. 
Theorem 3.10. Let $\left(X, p, \kappa_{1}\right)$ be an abelian digital H-group. If $\left(X, p, \kappa_{1}\right)$ and $\left(Y, q, \kappa_{2}\right)$ have the same $\left(\kappa_{1}, \kappa_{2}\right)$-homotopy type, then $\left(Y, q, \kappa_{2}\right)$ is an abelian digital H-group.

Proof. Let $f: X \rightarrow Y$ be a $\left(\kappa_{1}, \kappa_{2}\right)$-continuous function and let $g: Y \rightarrow X$ be a $\left(\kappa_{2}, \kappa_{1}\right)$-continuous function, such that $f \circ g \simeq_{\left(\kappa_{2}, \kappa_{2}\right)} 1_{Y}$ and $g \circ f \simeq_{\left(\kappa_{1}, \kappa_{1}\right)} 1_{X}$. Let $\mu$ and $\eta=f \circ \mu \circ(g \times g)$ be digital continuous multiplication of $\left(X, p, \kappa_{1}\right)$ and $\left(Y, q, \kappa_{2}\right)$, respectively. As $\mu$ is digital homotopy commutative, there exists a function $T: X \times X \rightarrow X \times X, T\left(x_{1}, x_{2}\right)=\left(x_{2}, x_{1}\right)$ such that $\mu \circ T \simeq_{\left(\kappa^{*}, \kappa_{1}\right)} \mu$. Now consider the function $T^{\prime}: Y \times Y \rightarrow Y \times Y, T^{\prime}\left(y_{1}, y_{2}\right)=\left(y_{2}, y_{1}\right)$. Then,

$$
\eta \circ T^{\prime}=f \circ \mu \circ(g \times g) \circ T^{\prime}=f \circ \mu \circ T \circ(g \times g) \simeq_{\left(\kappa^{*}, \kappa_{2}\right)} f \circ \mu \circ(g \times g)=\eta .
$$

So $\eta$ is digital homotopy commutative.

Proposition 1. [7] Let $\left(X, p, \kappa_{1}\right)$ be a digital image, $\left(Y, q, \kappa_{2}\right)$ be a digital H-group with digital continuous multiplication $\eta$. Then $\left[\left(X, p, \kappa_{1}\right),\left(Y, q, \kappa_{2}\right)\right]$ is a group under the product $[f] \bullet[g]=[\eta \circ(f \times g) \circ \triangle]$, for all $[f],[g] \in\left[\left(X, p, \kappa_{1}\right),\left(Y, q, \kappa_{2}\right)\right]$. Also if $\left(Y, q, \kappa_{2}\right)$ is an abelian digital H-group, then

$$
\left(\left[\left(X, p, \kappa_{1}\right),\left(Y, q, \kappa_{2}\right)\right], \bullet\right)
$$

is abelian.

Theorem 3.11. A homotopy associative digital $H$-space $(X, p, \kappa)$ is a digital $H$ group if and only if the map $\varphi: X \times X \rightarrow X \times X$ defined by $\varphi(x, y)=(x, x y)$ is a $\left(\kappa^{*}, \kappa^{*}\right)$-homotopy equivalence.

Proof. Let $(X, p, \kappa)$ be a digital H-group with digital homotopy inverse $\eta: X \rightarrow X$. Consider the digital continuous map $j: X \times X \rightarrow X \times X$ defined by $j(x, y)=$ $(x, \eta(x) y)$. Now $(\psi \circ j)(x, y)=\psi(x, \eta(x) y)=(x, x \varphi(x) y)$ implies that $\psi \circ j \simeq_{\left(\kappa^{*}, \kappa^{*}\right)}$ $1_{X \times X}$, since $\eta$ is the digital homotopy inverse. Also

$$
(j \circ \psi)(x, y)=j(x, x y)=(x, \eta(x) x y)
$$

implies $j \circ \psi \simeq \simeq_{\left(\kappa^{*}, \kappa^{*}\right)} 1_{X \times X}$. Hence $\psi$ is $\left(\kappa^{*}, \kappa^{*}\right)$ homotopy equivalence. Conversely, let $\Omega: X \times X \rightarrow X \times X$ be the digital homotopy inverse of $\psi$ such that $\psi \circ \Omega \simeq_{\left(\kappa^{*}, \kappa^{*}\right)}$ $\Omega \circ \psi \simeq \simeq_{\left(\kappa^{*}, \kappa^{*}\right)} 1_{X \times X}$. Now we go ahead to prove that $\left(X, p, \kappa_{1}\right)$ is a digital H-group. Define $\varphi: X \rightarrow X$ by the composite

$$
X \stackrel{i_{1}}{\rightarrow} X \times X \stackrel{\Omega}{\rightarrow} X \times X \stackrel{p_{2}}{\rightarrow} X .
$$

where $i_{1}: X \rightarrow X \times X$ is defined by $i_{1}(x)=(x, p)$ and $p i: X \times X \rightarrow X$ are the projections . Let $\mu: X \times X \rightarrow X$ be the digital continuous multiplication of $(X, p, \kappa)$, then $p_{1} \circ \psi=p_{1}, p_{2} \circ \psi=\mu$ and therefore

$$
\begin{aligned}
& p_{1} \simeq{ }_{\left(\kappa^{*}, \kappa\right)} p_{1} \circ \psi \circ \Omega=p_{1} \circ \Omega \\
& p_{2} \simeq{ }_{\left(\kappa^{*}, \kappa\right)} p_{2} \circ \psi \circ \Omega=\mu \circ \Omega .
\end{aligned}
$$

In particular, $p_{1} \circ \Omega \circ i_{1} \simeq_{(\kappa, \kappa)} p_{1} \circ i_{1}=1_{X}$. Hence

$$
\begin{aligned}
\mu \circ\left(1_{X}, \varphi\right) & =\mu \circ\left(p_{1} \circ \Omega \circ i_{1}, p_{2} \circ \Omega \circ i_{1}\right) \\
& =\mu \circ\left(p_{1}, p_{2}\right) \circ\left(\Omega \circ i_{1}\right) \\
& =\mu \circ \Omega \circ i_{1} \simeq_{(\kappa, \kappa)} p_{2} \circ i_{1}=c
\end{aligned}
$$

where $c: X \rightarrow X, \quad c(x)=p$ is the digital constant map. Similarly,

$$
\mu \circ\left(\varphi, 1_{X}\right) \simeq_{(\kappa, \kappa)} c .
$$


Hence $(X, p, \kappa)$ is a homotopy associative digital H-space such that $\varphi$ is the digital homotopy inverse. Consequently, $(X, p, \kappa)$ is a digital H-group.

Definition 3.12. [16] Let $\mathcal{C}$ and $\mathcal{D}$ be two categories. A contravariant functor $T$ from $\mathcal{C}$ to $\mathcal{D}$ is a mapping which associates to every object $X$ of $\mathcal{C}$ an object $T(X)$ of $\mathcal{D}$ and associates to every morphism $f: X \rightarrow Y$ of $C$ a morphism

$$
T(f): T(Y) \rightarrow T(X)
$$

of $D$ such that, $T\left(1_{X}\right)=1_{T(X)}$ and $T(g f)=T(f) T(g)$.

Theorem 3.13. [15] For any category $\mathcal{C}$ and object $Y$ of $\mathcal{C}$, there is a contravariant functor $\Pi^{Y}$ from $\mathcal{C}$ to the category of sets and functions which associates to an object $X$ of $\mathcal{C}$ the set $\Pi^{Y}(X)=\operatorname{hom}(X, Y)$ and to a morphism $f: X \rightarrow X^{\prime}$ the function $\Pi^{Y}(f)=f^{*}: \operatorname{hom}\left(X^{\prime}, Y\right) \rightarrow \operatorname{hom}(X, Y)$ defined by $f^{*}\left(g^{\prime}\right)=g^{\prime} \circ f$, for $g^{\prime}: X^{\prime} \rightarrow Y$.

Definition 3.14. The category whose objects are pointed digital images and the set of morphisms is $\operatorname{hom}\left(\left(X, p, \kappa_{1}\right),\left(Y, q, \kappa_{2}\right)\right)=\left[\left(X, p, \kappa_{1}\right),\left(Y, q, \kappa_{2}\right)\right]$ is called the homotopy category of the pointed digital images.

Theorem 3.15. Let $(X, p, \kappa)$ be a digital $H$-space with the digital continuous multiplication $\mu$ and $\left(Y, q, \kappa_{1}\right)$ and $\left(Z, r, \kappa_{2}\right)$ be any pointed digital images. Then there exists a homomorphism from $\left[\left(Z, r, \kappa_{2}\right),(X, p, \kappa)\right]$ to $\left[\left(Y, q, \kappa_{1}\right),(X, p, \kappa)\right]$.

Proof. Let $h:\left(Y, q, \kappa_{1}\right) \rightarrow\left(Z, r, \kappa_{2}\right)$ be a map and $[g],\left[g^{\prime}\right] \in\left[\left(Z, r, \kappa_{2}\right),(X, p, \kappa)\right]$. Let define $h^{*}:\left[\left(Z, r, \kappa_{2}\right),(X, p, \kappa)\right] \rightarrow\left[\left(Y, q, \kappa_{1}\right),(X, p, \kappa)\right]$ as $h^{*}([g])=[g \circ h]$. Then

$$
\begin{aligned}
h^{*}\left([g] \bullet\left[g^{\prime}\right]\right) & =h^{*}\left(\left[\mu \circ\left(g \times g^{\prime}\right) \circ \triangle\right]\right) \\
& =\left[\mu \circ\left(g \times g^{\prime}\right) \circ \triangle \circ h\right] \\
& =\left[\mu \circ\left((g \circ h) \times\left(g^{\prime} \circ h\right)\right) \circ \triangle\right] \\
& =[g \circ h] \bullet\left[g^{\prime} \circ h\right] \\
& =h^{*}([g]) \bullet h^{*}\left(\left[g^{\prime}\right]\right) .
\end{aligned}
$$

Therefore $\mathrm{h}^{*}$ is a homomorphism.

Theorem 3.16. Let $(Y, q, \kappa)$ be a digital H-group, then $\Pi^{Y}$ is a contravariant functor from the homotopy category of the pointed digital images to the category of groups and homomorphisms.

Proof. Let $\left(X, p, \kappa_{1}\right)$ and $\left(Z, r, \kappa_{2}\right)$ be objects and $[f] \in\left[\left(X, p, \kappa_{1}\right),\left(Z, r, \kappa_{2}\right)\right]$ is a morphism of the homotopy category of the pointed digital images.

$\Pi^{Y}\left(\left(X, p, \kappa_{1}\right)\right)=\operatorname{hom}\left(\left(X, p, \kappa_{1}\right),(Y, q, \kappa)\right)=\left[\left(X, p, \kappa_{1}\right),(Y, q, \kappa)\right]$.

Therefore $\Pi^{Y}\left(\left(X, p, \kappa_{1}\right)\right)$ is a group.

$$
\begin{aligned}
\Pi^{Y}([f]) & =f^{*}: \operatorname{hom}\left(\left(Z, r, \kappa_{2}\right),(Y, q, \kappa)\right) \rightarrow \operatorname{hom}\left(\left(X, p, \kappa_{1}\right),(Y, q, \kappa)\right) \\
& \Rightarrow f^{*}:\left[\left(Z, r, \kappa_{2}\right),(Y, q, \kappa)\right] \rightarrow\left[\left(X, p, \kappa_{1}\right),(Y, q, \kappa)\right]
\end{aligned}
$$

is a function defined as, $f^{*}([g])=[g \circ f]$, for any $[g] \in\left[\left(Z, r, \kappa_{2}\right),(Y, q, \kappa)\right]$. So, $f^{*}$ is a morphism between groups. Also by Theorem $19, f^{*}$ is a homomorphism.

Let show that $\Pi^{Y}$ is a contravariant functor. 
Let $[h] \in\left[\left(Z, r, \kappa_{2}\right) \rightarrow\left(W, t, \kappa_{3}\right)\right]$. For any morphism $\left[h^{\prime}\right] \in\left[\left(W, t, \kappa_{3}\right),(Y, q, \kappa)\right]$,

$$
\begin{aligned}
\Pi^{Y}([h])\left(\left[h^{\prime}\right]\right) & =\left[h^{\prime} \circ h\right] \\
\Pi^{Y}([f])\left(\left[h^{\prime} \circ h\right]\right) & =\left[\left(h^{\prime} \circ h\right) \circ f\right] \\
& =\left[h^{\prime} \circ(h \circ f)\right] \\
& =\Pi^{Y}([h \circ f])\left(\left[h^{\prime}\right]\right) .
\end{aligned}
$$

Thus,

$$
\Pi^{Y}([f])\left(\left[h^{\prime} \circ h\right]\right)=\Pi^{Y}([f])\left(\Pi^{Y}([h])\left(\left[h^{\prime}\right]\right)\right)=\left(\Pi^{Y}([f]) \circ \Pi^{Y}([h])\right)\left(\left[h^{\prime}\right]\right) .
$$

So, $\Pi^{Y}([h \circ f])=\Pi^{Y}([f]) \circ \Pi^{Y}([h])$.

Let $\left[1_{X}\right] \in\left[\left(X, p, \kappa_{1}\right),\left(X, p, \kappa_{1}\right)\right]$ be the unit morphism of the digital homotopy category of pointed digital images. Then,

$$
\Pi^{Y}\left(\left[1_{X}\right]\right)=1_{X}^{*}:\left[\left(X, p, \kappa_{1}\right),(Y, q, \kappa)\right], 1_{X}^{*}[(h)]=[h] .
$$

Consequently $\Pi^{Y}$ is a contravariant functor.

Corollary 3.17. Let $(Y, q, \kappa)$ be an abelian digital H-group, then $\Pi^{Y}$ is a contravariant functor from the homotopy category of the pointed digital images to the category of abelian groups and homomorphisms.

Theorem 3.18. Let $\left(Y, q, \kappa_{1}\right)$ and $\left(Z, r, \kappa_{2}\right)$ be digital $H$-spaces with the multiplications $\mu$ and $\eta$, respectiveliy, and $h:\left(Y, q, \kappa_{1}\right) \rightarrow\left(Z, r, \kappa_{2}\right)$ be a digital $H$ homomorphism. Then there exist a homomorphism from $\left[(X, p, \kappa),\left(Y, q, \kappa_{1}\right)\right]$ to $\left[(X, p, \kappa),\left(Z, r, \kappa_{2}\right)\right]$ for any pointed digital image $(X, p, \kappa)$.

Proof. Let $[g],\left[g^{\prime}\right] \in\left[(X, p, \kappa),\left(Y, q, \kappa_{1}\right)\right]$ and

$$
h_{*}:\left[(X, p, \kappa),\left(Y, q, \kappa_{1}\right)\right] \rightarrow\left[(X, p, \kappa),\left(Z, r, \kappa_{2}\right)\right]
$$

be a map such that $h_{*}([g])=[h \circ g]$. Then

$$
h_{*}\left([g] \bullet\left[g^{\prime}\right]\right)=h_{*}\left(\left[\mu \circ\left(g \times g^{\prime}\right) \circ \triangle\right]\right)=\left[h \circ \mu \circ\left(g \times g^{\prime}\right) \circ \triangle\right] .
$$

Since $h$ is a digital homomorphism, then $\eta \circ(h \times h) \simeq_{\left(\kappa^{*}, \kappa_{2}\right)} h \circ \mu$. So

$$
\begin{aligned}
{\left[h \circ \mu \circ\left(g \times g^{\prime}\right) \circ \triangle\right] } & =\left[\eta \circ(h \times h) \circ\left(g \times g^{\prime}\right) \circ \triangle\right] \\
& =[h \circ g] \bullet\left[h \circ g^{\prime}\right] \\
& =h_{*}([g]) \bullet h_{*}\left(\left[g^{\prime}\right]\right) .
\end{aligned}
$$

Therefore $h_{*}$ is a homomorphism.

Corollary 3.19. Let $(X, p, \kappa)$ be a digital $\mathrm{H}$-space and $\left(Y, q, \kappa_{1}\right)$ be a digital image. If $(X, p, \kappa)$ and $\left(Y, q, \kappa_{1}\right)$ have the same $\left(\kappa, \kappa_{1}\right)$-homotopy type, then there exist homotopy equivalences $f:(X, p, \kappa) \rightarrow\left(Y, q, \kappa_{1}\right)$ and $g:\left(Y, q, \kappa_{1}\right) \rightarrow(X, p, \kappa)$. For any digital image $\left(Z, r, \kappa_{2}\right)$

(1) since $f$ is a digital H-homomorphism, then there exist a homomorphism from $\left[\left(Z, r, \kappa_{2}\right),(X, p, \kappa)\right]$ to $\left[\left(Z, r, \kappa_{2}\right),\left(Y, q, \kappa_{1}\right)\right]$

(2) since $g$ is a digital H-homomorphism, then there exist a homomorphism from $\left[\left(Z, r, \kappa_{2}\right),\left(Y, q, \kappa_{1}\right)\right]$ to $\left[\left(Z, r, \kappa_{2}\right),(X, p, \kappa)\right]$. 


\section{REFERENCES}

[1] R. Ayala, E. Domínguez, A. R. Francés, and A. Quintero, Homotopy in digital spaces. Discrete Applied Mathematics, 125(1) , 3-24,(2003)

[2] L. Boxer, Digitally continuous functions, Pattern Recognition Letters 15.8, 833-839, (1994).

[3] L. Boxer, A classical construction for the digital fundamental group. Journal of Mathematical Imaging and Vision, 10(1), 51-62,(1999).

[4] L. Boxer, Properties of digital homotopy, Journal of Mathematical Imaging and Vision, 22(1), 19-26, (2005).

[5] O. Ege and I. Karaca, Digital H-spaces. In International Symposium on Computing in Science \& Engineering. Proceedings . GEDIZ University, Engineering and Architecture Faculty. p. 133, (2013, January).

[6] O. Ege and I. Karaca, Banach fixed point theorem for digital images. J. Nonlinear Sci. Appl, 8(3), 237-245, (2015).

[7] Ö. Ege and I. Karaca, Some properties of digital H-spaces. Turkish Journal of Electrical Engineering \& Computer Sciences, 24(3), 1930-1941, (2016).

[8] S. E. Han, Non-product property of the digital fundamental group. Information Sciences 171(13), 73-91,(2005).

[9] S. E. Han, Remarks on digital homotopy equivalence. Honam Mathematical Journal, 29(1), 101-118, (2007).

[10] S. E. Han, KD- $\left(\mathrm{k}_{0}, \mathrm{k}_{1}\right)$-homotopy equivalence and its applications. Journal of the Korean Mathematical Society, 47(5), 1031-1054, (2010).

[11] T. Y: Kong, Roscoe, A. W. and Rosenfeld, A., Concepts of digital topology. Topology and its Applications, 46(3), 219-262, (1992).

[12] A. Mutlu, B. Mutlu and S. Öztunç, On Digital Homotopy of Digital Paths, International Research Journal of Pure Algebra 2(6), 147-154, (2012).

[13] S. Öztunç, S. and Mutlu, A. Categories in digital images. Am. J. Math. Stat, 3(1), 62-66, (2013).

[14] A. Rosenfeld, Digital topology, The American Mathematical Monthly, 86(8)621-630, (1979).

[15] R. M. Switzer, Algebraic topology-homotopy and homology, Springer. (2017).

[16] E. H. Spanier, Algebraic topology, Springer Science \& Business Media 55(1) (1989).

[17] T. Yung Kong, A digital fundamental group, Computers \& Graphics 13.2, 159-166,(1989)

(author one) Kastamonu University, Department of Mathematics, Kastamonu, Turkey E-mail address, author one: sdemiralp@kastamonu.edu.tr

(author two) Beni-Suef University, Department of Basic Science, Faculty of IndusTRIAL EducATion, Egypt

E-mail address, author two: essam.salim@techedu.bsu.edu.eg 